MIXED FINITE ELEMENT METHODS FOR

COMPUTING GROUNDWATER VELOCITIES

M.B. Allen

R.E. Ewing

J.V. Koebbe

1985

Journal Article

WWRC $-85-28$

In

Numerical Methods for Partial

Differential Equations

Volume 3

\author{
M.B. Allen \\ R.E. Ewing \\ J.V. Koebbe \\ Department of Mathematics \\ University of Wyoming \\ Laramie, Wyoming
}




\title{
Mixed Finite Element Methods for Computing Groundwater Velocities
}

\author{
M. B. Allen, R. E. Ewing, and J.V. Koebbe \\ Department of Mathematics, University of Wyoming, \\ Laramie, Wyoming 82171 \\ Central to the understanding of problems in water quality and quantity for effective \\ management of water resources is the development of accurate numerical models to \\ stimulate groundwater flows and contaminant transfer. We discuss several important dif- \\ ficulties arising in modeling of subsurface flow and present promising numerical proce- \\ dures for alleviating these problems. Furthermore, we describe mixed-finite element \\ techniques for accurately approximating fluid velocities, and review computational re- \\ sults on a variety of hydrologic problems.
}

\section{INTRODUCTION}

In the past decade, water quality problems have assumed increasing importance in water resources engineering. An emerging awareness that our groundwater supplies face the threat of contamination from various sources has prompted vigorous research into mathematical methods for predicting contaminant movements in underground water. In many respects the task of simulating contaminant flows in porous media is computationally more demanding than the more traditional problem of resolving supply issues. The fundamental reason for this increased difficulty is that in contaminant flows, the fluid velocity plays a crucial role, while water supply problems more typically concern a scalar field such as head or pressure. According to Darcy's law, one must differentiate heads or pressures to get velocities, and this leads to at least two related mathematical problems. First, any pathologic behavior in pressure or head manifests itself in even more severe behavior in velocity. Thus, for example, the relatively mild logarithmic singularities in pressure or head that occur at pumped wells appear as simple poles in the velocity field. Second, standard numerical solutions of the flow equations commonly produce discrete approximations to the pressure or head, and in differentiating these approximations to compute velocities one incurs a loss of accuracy that is typically one order in the spatial grid mesh.

These difficulties are significant, for they can lead, in the first case, to nonconvergent approximations to velocities near wells and, in the second case, to inferior predictions of the very aspect of groundwater motion that is most crucial in forecasting contaminant transport. In this paper we examine a mixed finite element method for the groundwater flow equations that mitigates these difficulties. The essential idea of the mixed method is that, by solving the second-order equation governing groundwater flow as a set of coupled firstorder equations in velocity and head, one can compute both fields explicitly without sacrificing accuracy in the velocity through differentiation. The method

Numerical Methods for Partial Differential Equations, 3, 195-207 (1985)

(C) 1985 John Wiley \& Sons, Inc.

CCC 0749-159X/85/030195-13\$04.00 
also admits natural choices of interpolating polynomials for the trial functions to guarantee the highest accuracy for a given number of degrees of freedom. Furthermore, in problems involving pumped wells, one can incorporate appropriate singularities in the trial functions for velocity. The singular parts, being known, then contribute to the inhomogeneous terms in the systems of algebraic equations that arise through spatial discretization. This approach circumvents convergence difficulties near wells and leads to good global error estimates.

Proper choice of trial functions is an essential feature of the mixed method presented here. In particular, the trial space used for the fluid velocity must yield an approximation whose divergence lies in the trial space for head; otherwise the favorable convergence rates cited below are no longer valid. Thus, the methods advanced here are distinct from other formulations that also treat velocity and head as principal unknowns but use trial functions belonging to the same continuity class for both [1].

\section{REVIEW OF THE MIXED METHOD}

Let us examine a model equation arising in the simulation of steady-state flow in a two-dimensional, horizontal, leaky aquifier. This type of problem is representative of the sorts of flow equations that need to be solved in conjunction with species transport equations in groundwater contamination studies. The governing equation is

$$
\nabla \cdot(T \nabla h)-\frac{K}{b}\left(h-h_{a}\right)+Q=0
$$

where $h$ is the unknown head in the aquifier, $T$ is the transmissivity, $K$ is the hydraulic conductivity in the aquitard overlying the leaky aquifier, $b$ is the thickness of the aquitard, $h_{a}$ is the head in the aquitard, and $Q$ represents internal sources or sinks. If the sources or sinks are all wells, then we can idealize them as points:

$$
Q=\sum_{\ell=1}^{L} Q_{\ell} \delta\left(\mathbf{x}-\mathbf{x}_{\ell}\right)
$$

Here $Q_{\ell}$ stands for the strength of the $\ell$-th source (negative for producing wells), and $\delta\left(\mathbf{x}-\mathbf{x}_{\ell}\right)$ is the Dirac distribution centered at spatial position $\mathbf{x}_{\ell}$. The leakage term in (1) has the linear form proposed by Charbeneau and Street [2].

In the mixed finite-element method we factor Darcy's law from Eq. (1), giving a coupled set of first-order equations:

$$
\begin{gathered}
\mathbf{u}+T \nabla h=0 \\
-\nabla \cdot \mathbf{u}-\frac{K}{b}\left(h-h_{a}\right)+Q=0
\end{gathered}
$$

where $\mathbf{u}$ is the superficial or Darcy velocity of the water. In typical boundaryvalue problems we solve Eqs. (2) on a bounded open set $\Omega \subset \mathrm{IR}^{2}$ subject to boundary data of the form 


$$
\begin{array}{cc}
\mathbf{u}(\mathbf{x}) \cdot \boldsymbol{v}(\mathbf{x})=0, & \mathbf{x} \in \partial \Omega_{N} \\
h(\mathbf{x})=h_{\partial}(\mathbf{x}), & \mathbf{x} \in \partial \Omega_{D} .
\end{array}
$$

Thus the orientable boundary $\partial \Omega$, having unit outward normal vector $\boldsymbol{v}$, admits a decomposition $\partial \Omega_{N} \cup \partial \Omega_{D}$ into no-flow and prescribed-head segments. $\partial \Omega_{N}$ is a locus of points where normal fluid velocities vanish, while $\partial \Omega_{D}$ is the boundary segment along which heads are known.

The boundary-value problem formed by Eqs. (2) and (3) has a variational form that underlies the finite-element approximations. Let $L^{2}(\Omega)$ be the space of square-integrable functions on $\Omega$, and define the trial spaces:

$$
V=\left\{\mathbf{v} \in L^{2}(\Omega) \times L^{2}(\Omega) \mid \nabla \cdot \mathbf{v} \in L^{2}(\Omega) \text { and } \mathbf{v} \cdot v=0 \text { on } \partial \Omega_{N}\right\}
$$

being the space of vector-valued velocity trial functions, and

$$
W=\left\{w \in L^{2}(\Omega) \mid w=h_{\partial} \text { on } \partial \Omega_{D}\right\}
$$

being the space of trial functions for the head. Observe that functions belonging to $V$ not only obey the no-flux boundary conditions but also have divergences lying in $L^{2}(\Omega)$. This inclusion, which is a natural mathematical feature of the problem, must be preserved in discrete analogs to ensure good error estimates. The variational version of our boundary-value problem is a set of integral equations obtained using the inner products $(f, g)=\int_{\Omega} f g d v$ and $(\mathbf{f}, \mathbf{g})=\int_{\Omega} \mathbf{f} \cdot \mathbf{g} d v$ : we seek $\mathbf{u} \in V$ and $h \in W$ such that

$$
\begin{gathered}
\left(T^{-1} \mathbf{u}+\nabla h, \mathbf{v}\right)=0 \text { for all } \mathbf{v} \in V \\
\left(-\nabla \cdot \mathbf{u}-\frac{K}{b}\left(h-h_{a}\right)+Q, w\right)=0 \quad \text { for all } w \in W .
\end{gathered}
$$

Integrating by parts and observing the boundary values of the trial functions gives

$$
\begin{gathered}
\left(T^{-1} \mathbf{u}, \mathbf{v}\right)-(h, \nabla \cdot \mathbf{v})=-\int_{\partial \Omega_{D}} h \mathbf{v} \cdot \mathbf{v} d s \quad \text { for all } \mathbf{v} \in V \\
(\nabla \cdot \mathbf{u}, w)+\left(\frac{K}{b} h, w\right)=\left(\frac{K}{b} h_{a}+Q, w\right) \quad \text { for all } w \in W .
\end{gathered}
$$

Finite-element approximations to the boundary value problem given in Eqs. (2) and (3) are analogs of Eqs. (4) posed on finite-dimensional subspaces $V_{k}$ and $W_{k}$ of the trial spaces $V$ and $W$. In particular, we choose subspaces of piecewise polynomial interpolating functions on $\Omega$. The index $k$ therefore indicates the mesh of partitions for finite-element interpolation.

To define the specific subspaces used in this paper, we need to introduce some notation. For simplicity let us choose $\Omega$ to be a rectangle, $\Omega=I \times J$, where $I=(a, b)$ and $J=(c, d)$ are open intervals in $x$ and $y$, respectively. Consider partitions $\Delta_{x}: a=x_{0}<\cdots<x_{M}=b$ and $\Delta_{y}: c=y_{0}<\cdots<$ $y_{N}=d$ of $I$ and $J$ having mesh:

$$
k=\max _{\substack{1 \leq i \leq M \\ 1 \leq j \leq N}}\left\{x_{i}-x_{i-1}, y_{j}-y_{j-1}\right\} .
$$


We define piecewise polynomial space $M_{q}^{p}$ on a given partition $\Delta$ of any interval $S$ to be the space of $q$-times continuously differentiable functions that, when restricted to a single interval in the partition, reduce to polynomials of degree not greater than $p: M_{q}^{p}(\Delta)=\left\{\psi \in C^{q} \mid \psi\right.$ is a polynomial of degree at most $p$ on each subinterval of $\Delta\}$. Thus, for example, $M_{-1}^{0}$ is a space of piecewise constant functions that may be discontinuous between subintervals, while $M_{0}^{1}$ is a space of continuous, piecewise linear functions.

For our trial spaces, we choose tensor-product Raviart-Thomas [3] subspaces on the rectangle $I \times J$. In the lowest-degree case, we pick

$$
\begin{gathered}
W_{k}=\left\{w_{k} \in M_{-1}^{0}\left(\Delta_{x}\right) \otimes M_{-1}^{0}\left(\Delta_{y}\right) \mid w_{k}=h_{\partial} \text { on } \partial \Omega_{D}\right\} \\
V_{k}=\left\{\mathbf{v}_{k} \in\left[M_{0}^{1}\left(\Delta_{x}\right) \otimes M_{-1}^{0}\left(\Delta_{y}\right)\right] \times\left[M_{-1}^{0}\left(\Delta_{x}\right) \otimes M_{0}^{1}\left(\Delta_{y}\right)\right] \mid \mathbf{v}_{k} \cdot \boldsymbol{v}=0 \text { on } \partial \Omega_{N}\right\} .
\end{gathered}
$$

In this case our trial function for the head $h$ will be piecewise constant in the $x$ and $y$ directions. The trial function for velocity $\mathbf{u}$ will have two components: the $x$-component will be piecewise linear and continuous in the $x$ direction and piecewise constant with jump discontinuities in the $y$ direction, while the $y$ component will be piecewise constant in $x$ and piecewise linear in $y$. For the next highest degree of approximation we choose

$$
\begin{gathered}
\dot{W}_{k}=\left\{w_{k} \in M_{-1}^{1}\left(\Delta_{x}\right) \otimes M_{-1}^{1}\left(\Delta_{y}\right) \mid w k=h_{\partial} \text { on } \partial \Omega_{D}\right\} \\
V_{k}=\left\{\mathbf{v}_{k} \in\left[M_{0}^{2}\left(\Delta_{x}\right) \otimes M_{-1}^{1}\left(\Delta_{y}\right)\right] \times\left[M_{-1}^{1}\left(\Delta_{x}\right) \otimes M_{0}^{2}\left(\Delta_{y}\right)\right] \mid \mathbf{v}_{k} \cdot \boldsymbol{v}=0 \text { on } \partial \Omega_{N}\right\} .
\end{gathered}
$$

Notice that the degrees of the polynomials have increased by 1 over the firstorder spaces, but the degrees of continuity remain the same.

Having chosen our trial spaces, we derive finite-element analogs of Eqs. (4) by forming trial functions $\hat{h}_{k} \in W_{k}$ and $\hat{\mathbf{u}}_{k} \in V_{k}$ whose values at the nodes $\left(x_{i}, y_{j}\right)$ of the partition $\Delta_{x} \times \Delta_{y}$ are unknown. To solve for these unknown coefficients, we impose the Galerkin criteria:

$$
\begin{aligned}
& \left(T^{-1} \hat{\mathbf{u}}, \mathbf{v}_{k}\right)-\left(h_{k}, \nabla \cdot \mathbf{v}_{k}\right)=-\int_{\partial \Omega_{0}} h_{k} \mathbf{v}_{k} \cdot v d s \quad \text { for all } \mathbf{v}_{k} \in V_{k} \\
& \left(\nabla \cdot \mathbf{u}_{k}, w_{k}\right)+\left(\frac{K}{b} h_{k}, w_{k}\right)=\left(\frac{K}{b} h_{a}+Q, w_{k}\right) \text { for all } w_{k} \in W_{k} .
\end{aligned}
$$

These equations are just finite-dimensional analogs of the variational equations derived earlier.

In problems having pumped wells in $\Omega$ the velocity field will possess poles of order one. Error estimates relying on smoothness in the approximated solution fail near these singularities, and as a result many standard finite-element approximations to fluid velocity do not converge near wells. To avoid poor polynomial approximations near wells we modify the trial function for the velocity to accommodate the singularities. Hence, we decompose $\hat{\mathbf{u}}_{k}$ into a regular part and a singular part: $\hat{\mathbf{u}}_{k}=\hat{\mathbf{u}}_{r}+\hat{\mathbf{u}}_{s}$. Since we know the strengths, locations, and local forms of the singularities, we can write 


$$
\hat{\mathbf{u}}_{s}=\frac{1}{2 \pi} \sum_{l=1}^{L} Q_{\ell} \nabla \log \left|\mathbf{x}-\mathbf{x}_{\ell}\right|
$$

and therefore treat $\hat{\mathbf{u}}_{s}$ as known. In this case Eqs. (5) become

$$
\left(T^{-1} \hat{\mathbf{u}}_{r}, \mathbf{v}_{k}\right)-\left(h_{k}, \nabla \cdot \mathbf{v}_{k}\right)=\int_{\partial \Omega_{0}} h_{k} \mathbf{v}_{k} \cdot \boldsymbol{v} d s-\left(T^{-1} \hat{\mathbf{u}}_{s}, \mathbf{v}_{k}\right) \text { for all } \mathbf{v}_{k} \in V_{k}
$$

and

$$
\begin{aligned}
\left(\nabla \cdot \hat{\mathbf{u}}_{r}, w_{k}\right)+\left(\frac{K}{b} h_{k}, w_{k}\right)= & \left(\frac{K}{b} h_{a}+Q, w_{k}\right) \\
& -\left(\nabla \cdot \hat{\mathbf{u}}_{s}, w_{k}\right) \text { for all } w_{k} \in W_{k} .
\end{aligned}
$$

Evaluating the integrals appearing in these equations leads to a set of linear algebraic equations in the unknown nodal coefficients of $\hat{\mathbf{u}}_{r}$ and $\hat{h}$.

\section{THEORY}

As mentioned earlier, the class of methods just described has two advantages over traditional finite-element formulations: they retain high-order accuracy in the velocities by obviating differentiation, and they eliminate convergence difficulties near wells through the subtraction of singularities from trial func' tions. These advantages have their bases in theoretical error estimates. For the more traditional, straightforward projections of the variational analog of Eq. (1) onto interpolating subspaces, fluid velocities must be computed from heads as $\mathbf{u}=-T \nabla h$. Standard approximation theory [4] reveals that a piecewise polynomial method furnishing $O\left(k^{\prime}\right)$ approximations to $h$ yields approximations to $\nabla h$ that are only $O\left(k^{r-1}\right)$ as $k \rightarrow 0$. Thus improvements in the accuracy of $\mathbf{u}$ require greater refinement of the finite-element partition than comparable improvements in the accuracy of $h$. In contrast, the mixed method suffers no such disparity. Douglas, Ewing, and Wheeler [5] show that, in regions where the source term $Q$ is smooth, the mixed method using the first- and second-order trial spaces described above has global error bounds of the form

$$
\begin{aligned}
\|\hat{\mathbf{u}}-\mathbf{u}\|_{2} & \leq M_{1} k \\
\|\hat{h}-h\|_{2} & \leq M_{2} k
\end{aligned}
$$

and

$$
\begin{aligned}
\|\hat{\mathbf{u}}-\mathbf{u}\|_{2} & \leq M_{3} k^{2} \\
\|\hat{h}-h\|_{2} & \leq M_{4} k^{2}
\end{aligned}
$$

respectively, where $M_{1}, M_{2}, M_{3}, M_{4}$ are constants for a given boundary value problem and $\|\cdot\|_{2}$ signifies the norm associated with the inner product $(\cdot, \cdot)$. Thus refining the spatial partition in the mixed method yields comparable improve- 
ments in both heads and velocities. As Douglas, Ewing, and Wheeler [5] demonstrate, however, the inclusion relationship between the divergence of the velocity trial space and the head trial space is an essential fact in deriving these error estimates.

The error estimates have implications for problems involving nonhomogeneous media. In standard formulations with spatially heterogeneous transmissivities the calculation $\mathbf{u}=-T \nabla h$ calls for the multiplication of a function, $T$, that may be rapidly varying for physical reasons, with another, $\nabla h$, that may vary rapidly simply by virtue of its being the gradient of a spatially varying approximation. Such a product of rapidly varying functions may be quite poorly behaved in numerical models. The mixed method avoids the numerical noise associated with differentiation of heads and therefore does not compound physical fluctuations with artificial ones.

Douglas, Ewing, and Wheeler [5] also give theoretical justification to the subtraction of singularities. In this case both the first- and second-order schemes give global error estimates of the form

$$
\begin{aligned}
\|\hat{\mathbf{u}}-\mathbf{u}\|_{2} & \leq M_{5} k \log \left(k^{-1}\right) \\
\|\hat{h}-h\|_{2} & \leq M_{6} k \log \left(k^{-1}\right)
\end{aligned}
$$

where, again, $M_{5}$ and $M_{6}$ are constants for a given boundary-value problem. These estimates ensure that the velocities predicted by the mixed method will converge to the exact velocities near pumped wells when the trial function $\hat{\mathbf{u}}$ explicitly incorporates simple poles at the wells.

\section{COMPUTATIONAL EXAMPLE}

To illustrate the effectiveness of the mixed method we shall examine a simple numerical example. Consider the equation

$$
\nabla^{2} h-(h-1)+Q=0
$$

on $\Omega=(0,1) \times(0,1)$ with $Q=\delta(\mathbf{x}-(1,1))$ and $\mathbf{u} \cdot \boldsymbol{\nu}=0$ on $\partial \Omega$. We shall examine various pressure and velocity solutions for this boundaryvalue problem.

Before discussing the numerical results, however, it is worth reviewing our choice of bases for the trial spaces $V_{k}$ and $W_{k}$. For convenience let us temporarily use the variable $z$ to stand for either $x$ or $y$, let the partition in the $z$ direction be $\Delta_{z}: z_{0}<\cdots<z_{\Lambda}$, and call $\Delta z_{\lambda}=z_{\lambda}-z_{\lambda-1}, \lambda=1, \ldots, \Lambda$. Define the functions $\left\{v_{\gamma}\right\}_{\gamma=1}^{2 \Lambda}=1$ as follows. If $\gamma$ is even, $v_{\gamma}$ is the standard piecewise linear chapeau function having $v_{\gamma}\left(z_{\beta}\right)=\delta_{\gamma \beta}$. If $\gamma$ is odd, say $\gamma=$ $2 \lambda-1$, then $v_{\gamma}$ is the piecewise quadratic given by

$$
v_{2 \lambda-1}^{(z)}=\left\{\begin{array}{l}
4\left(z-z_{\lambda-1}\right)\left(z_{\lambda}-z\right) /(\Delta z)^{2}, \quad z \in\left[z_{\lambda-1}, z_{\lambda}\right] . \\
0, \quad \text { otherwise } .
\end{array}\right.
$$


Now take $M_{-1}^{1}\left(\Delta_{z}\right)=\operatorname{span}\left\{v_{\gamma}\right\}_{\gamma=1}^{2 \Lambda}$. To get a basis for $M_{0}^{2}\left(\Delta_{z}\right)$ define the functions $\left\{w_{\lambda 0}, w_{\lambda 1}\right\}_{\lambda=1}^{\Lambda}$ as follows:

$$
\begin{aligned}
& w_{\lambda 0}(z)= \begin{cases}\frac{z_{\lambda-1}+\sigma_{2} \Delta z_{\lambda}-z}{\left(\sigma_{2}-\sigma_{1}\right) \Delta z_{\lambda}}, & z \in\left(z_{\lambda-1}, z_{\lambda}\right) \\
0, \quad \text { otherwise }\end{cases} \\
& w_{\lambda 1}(z)= \begin{cases}\frac{z-z_{\lambda-1}-\sigma_{1} \Delta z_{\lambda}}{\left(\sigma_{2}-\sigma_{1}\right) \Delta z_{\lambda}}, & z \in\left(z_{\lambda-1}, z_{\lambda}\right) \\
0, \quad \text { otherwise }\end{cases}
\end{aligned}
$$

where $\sigma_{1}, \sigma_{2}$ are the Gauss points $\left(1 \pm \sqrt{3}^{-1}\right) / 2$ in the unit interval $(0,1)$. Then $M_{0}^{2}=\operatorname{span}\left\{w_{\lambda 0}, w_{\lambda 1}\right\}_{\lambda=1}^{\Lambda}$. With these definitions, we can form tensorproduct bases for the spaces $W_{k}$ and $V_{k}$ introduced in Section II.

Using these bases we can compute the matrix equation representing the discrete Galerkin approximation to the model problem. It happens that, while the matrix is sparse, positive-definite, and invertible, it is not particularly well

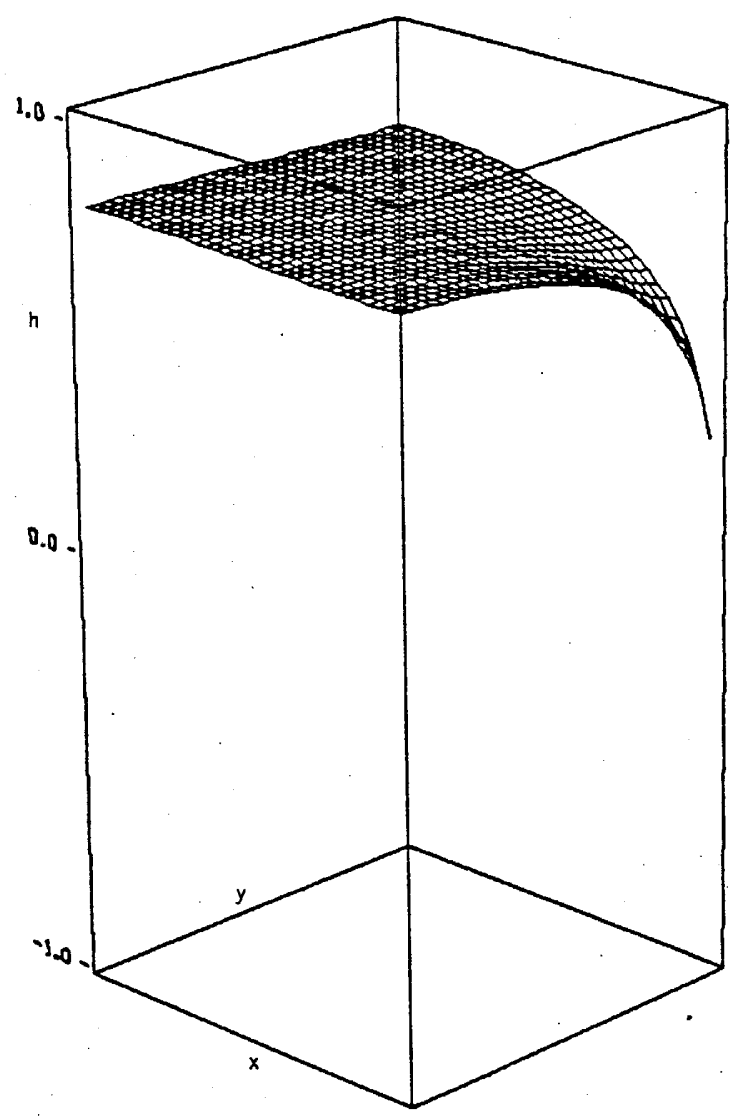

FIG. 1. Head distribution computed using first-order elements on a square grid having 32 elements on a side. 


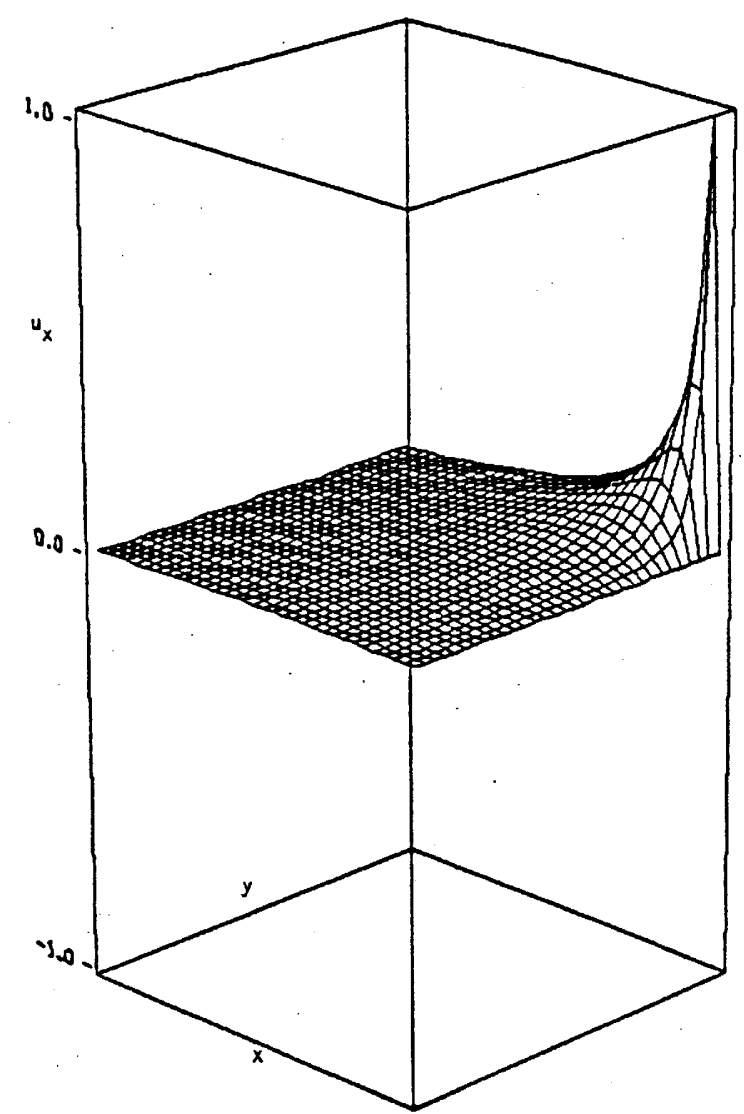

FIG. 2. $x$-velocity distribution computed using first-order elements on a square grid having 32 elements on a side.

conditioned. Ewing and Koebbe [6] describe an application of preconditioned conjugate-gradient techniques to overcome the poor conditioning and speed the iterative solution of the linear system.

Figure 1 shows the pressure or head distribution over $\Omega$ computed using the lowest degree (first-order) elements on a square grid having 32 elements on a side. Figure 2 shows the corresponding field for the $x$-component of water velocity. These solutions exhibit a logarithmic drawdown in head near the producing well together with a concomitant pole in $u_{x}$. Figures 3 and 4 shows the head and $x$-velocity distributions computed for the same problem using the secondorder trial space on a square grid having 16 elements on a side. Since the second-order method requires approximately twice as many degrees of freedom per element in each coordinate direction, the number of nodal unknowns needed to generate Figures 3 and 4 is comparable to the number needed in Figures 1 and 2. The two pairs of plots are quite similar, as one might expect considering the parity in computational effort between the two cases.

The method also performs well in problems with heterogeneous medium properties. Figure 5, for example, shows the $x$-velocity distribution that results 


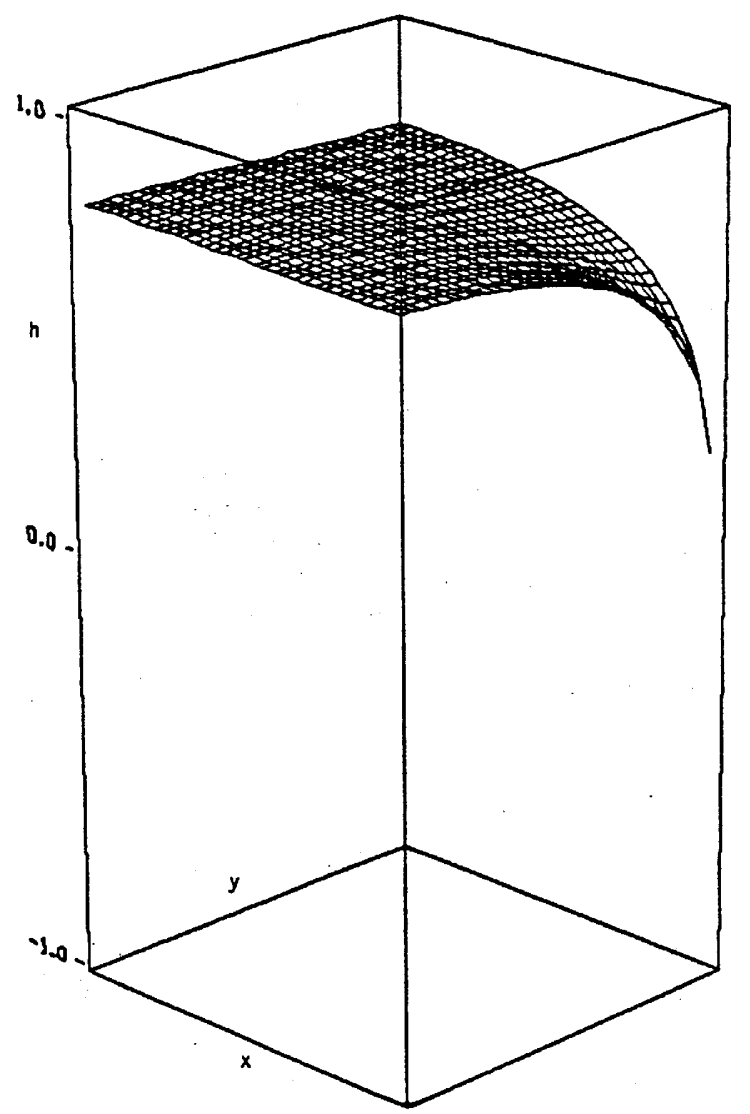

FIG. 3. Head distribution computed using second-order elements on a square grid having 16 elements on a side.

when we use first-order elements and impose a nonuniform transmissivity having the form

$$
T(x, y)= \begin{cases}1, & y \geq x \\ 0.01, & y<x .\end{cases}
$$

Thus $T$ suffers a jump discontinuity along a line running diagonally through $\Omega$ into the wellbore. The $x$-velocity away from the wellbore therefore remains small for $y<x$ but increases rapidly toward the wellbore near the edge of the domain where $y=1$.

Figure 6 illustrates the $x$-velocity that results from using second-order elements and a discontinuous aquitard head $h_{a}$ of the form

$$
h_{a}(x, y)=\left\{\begin{array}{l}
1.0, \quad x \leq 0.5 \\
0.01, \quad x>0.5 .
\end{array}\right.
$$

The contour plot of $u_{x}$ in Figure 7 shows more clearly the radial flow dominant near the well and the "ridgeline" pattern prevailing away from the well. While 


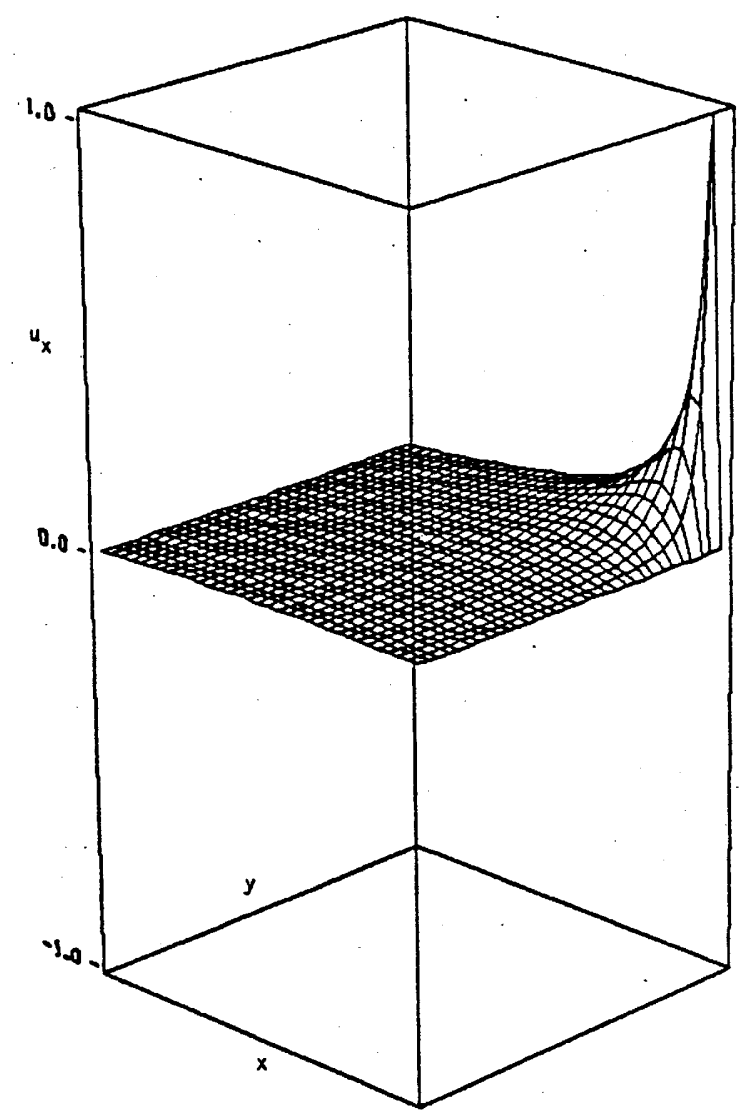

FIG. 4. $x$-velocity distribution computed using second-order elements on a square grid having 16 elements on a side.

heterogeneities of the forms given in Eqs. (7) and (8) are highly idealized, they provide simple yet relatively strenuous tests of the mixed method's ability to model problems with nonuniform material properties.

\section{CONCLUSIONS}

We have seen that the mixed finite-element method is an attractive approach for solving groundwater flow equations, especially in contaminant transport problems where accurate water velocities are paramount. The method gives velocities that have the same order of accuracy as heads, affording rapid error reductions on grid refinement compared with the traditional finite-element approach. Further advantages accrue through the explicit incorporation of source and sink singularities in the trial functions for velocity. Here the improvement over traditional discrete methods is more dramatic: the mixed method with subtracted singularities converges at wells, while traditional schemes do not. 


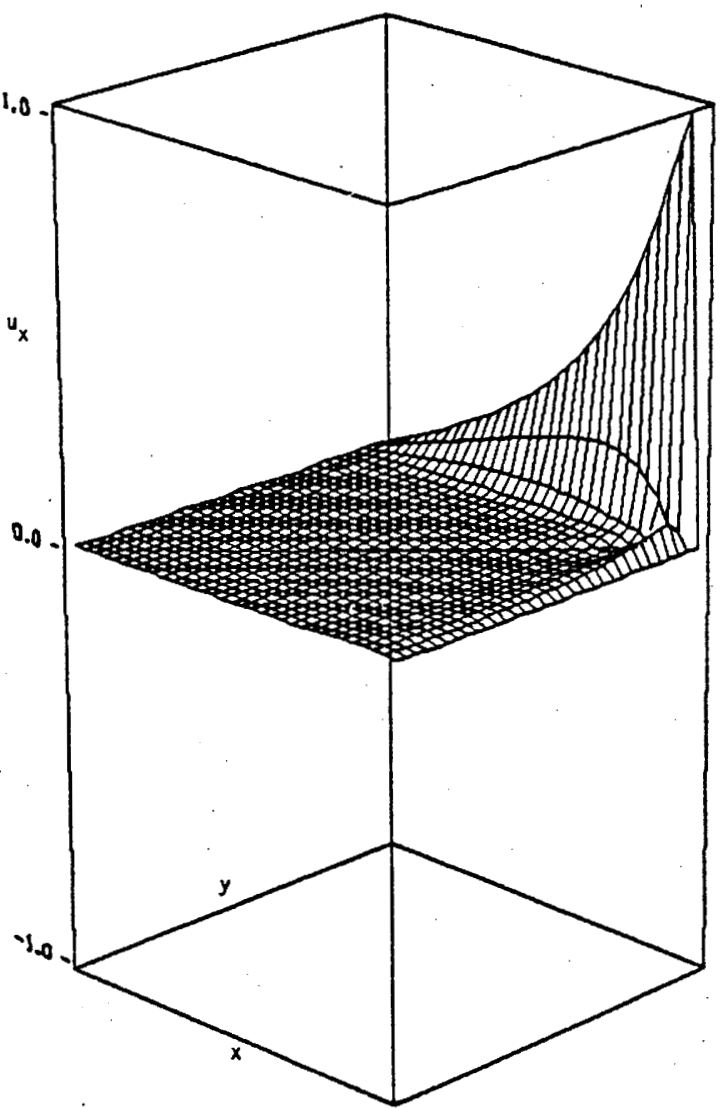

FIG. 5. $x$-velocity distribution computed using first-order elements on a problem in which the transmissivity has a discontinuity along $y=x$.

Finally, the mixed method gives good numerical results even in problems with rather severe heterogeneities in medium properties.

This research is supported in part by Contract No. DAAG29-84-K-002 from the Army Research Office, by Grant No. CEE-8404266 from the National Science Foundation, and by a grant from the Wyoming Water Research Center.

\section{References}

[1] G. Segol and G. F. Pinder, "Transient simulation of saltwater intrusion in southeastern Florida." Water Res. Research 23:65-70 (1976).

[2] R. J. Charbeneau and R. L. Street, "Modeling groundwater flow fields containing point singularities: a technique for singularity removal." Water Res. Research 15:583-599 (1979).

[3] P. A. Raviart and J. M. Thomas, A Mixed Finite Element Method for Second-Order Elliptic Problems: Mathematical Aspects of the Finite Element Method. SpringerVerlag, Heidelberg, 1977. 


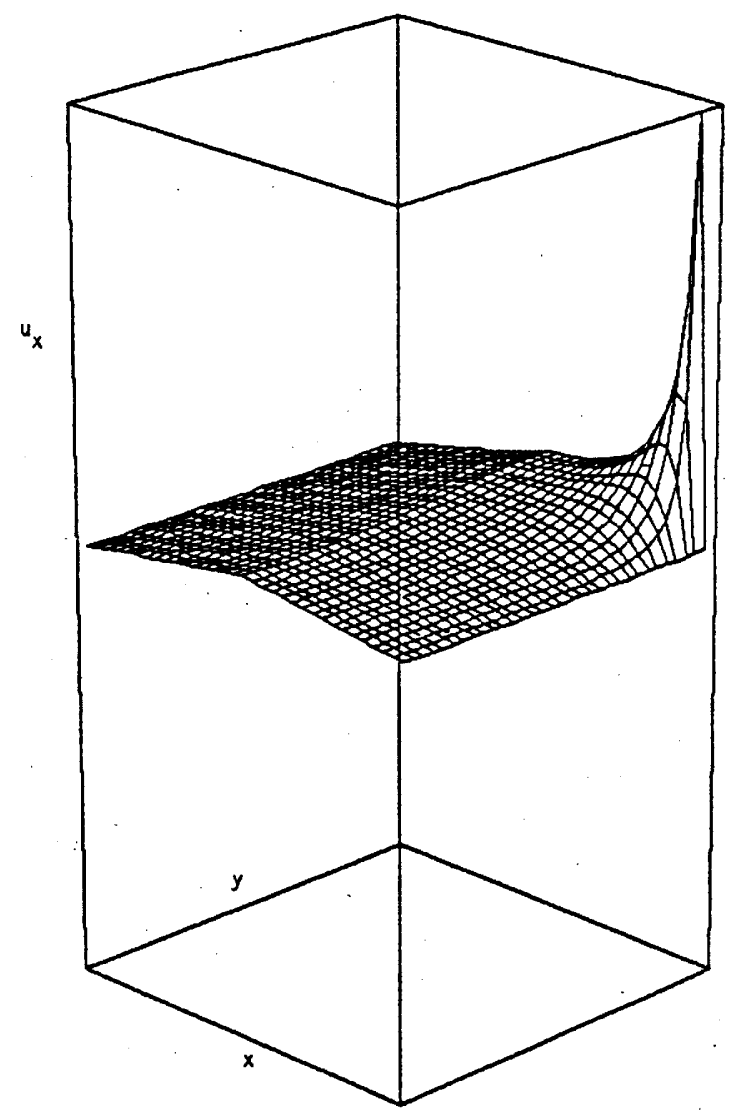

FIG. 6. $x$-velocity distribution computed using second-order elements on a problem where the acquitard head $h_{a}$ has a discontinuity atong $x=0.5$.

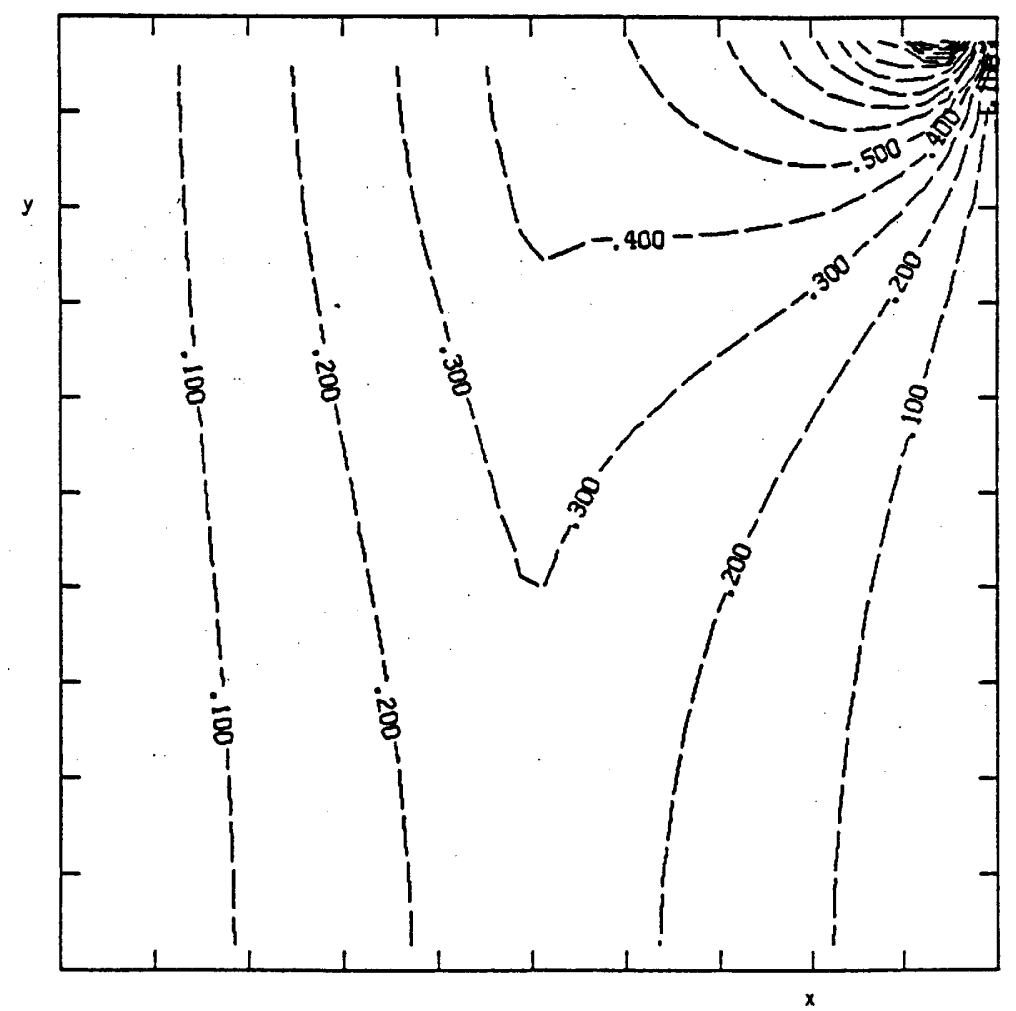

FIG. 7. Contour plot of $x$-velocities shown in Figure 6. 
[4] P. M. Prenter, Splines and Variational Methods. John Wiley \& Sons, New York, 1975.

[5] J. Douglas, R.E. Ewing, and M.F. Wheeler, "The approximation of the pressure by a mixed method in the simulation of miscible displacement." RAIRO Analyse Numerique 17:17-34 (1983).

[6] R.E. Ewing and J. V. Koebbe, "Mixed finite element methods for groundwater flow and contaminant transport," in Proceedings of the Fifth IMACS International Symposium on Computer Methods for Partial Differential Equations, Bethlehem, Pennsylvania, June 19-21, 1984, p. 106-113. 